«Un rêve impossible à réaliser, nécessairement déçu lorsqu'on travaille en solitaire, mais qui semble plus que jamais fonder la seule méthode pour faire avancer les questions, même si une telle

méthode rend la grande thèse de plus en plus impraticable et le travail d'équipe indispensable » CARRIERE (1987) p. 5.

\title{
Sciences de l'éducation, sciences de la formation et renouvellement des humanités
}

Richard Étienne Professeur d'université émérite en sciences de l'éducation Université Paul Valéry Montpellier - France Laboratoire LIRDEF-TFD, équipe d'accueil 3749 rietienne@wanadoo.fr

\section{Introduction}

L'écriture de ce chapitre d'un ouvrage de mélanges offert à Ghislaine Viré m'incite à rendre également un vibrant hommage à un ardent défenseur des langues et civilisations anciennes, trop tôt disparu, qui a pensé que le voyage et la culture faisaient partie du patrimoine de l'humanité. Bernard Valette, professeur de lettres au lycée d'Alès et fondateur de l'association culturelle Thalassa a fait découvrir les lieux et paysages du monde antique à des dizaines de milliers de jeunes élèves. Il aimait à reprendre la formule de Térence « Rien de ce qui est humain ne m'est étranger ». Je souhaiterais m'inscrire dans la trace de ses pas pour écrire ces pages et montrer en quoi les sciences de l'éducation font partie des humanités d'aujourd'hui qui nous réunissent et contribuent au renouvellement des méthodes et des enseignements. Ayant été un didacticien des langues anciennes ${ }^{1}$ avant que de devenir un chercheur sur le changement en éducation, je ne parlerai donc que de ce que je connais... un peu !

Je commencerai par évoquer les liens paradoxaux qu'entretiennent deux disciplines aussi contrastées que les langues et cultures de l'antiquité (LCA), appellation française actuelle, et les toutes récentes sciences de l'éducation ${ }^{2}$ dont quelques esprits mal intentionnés font leur cible privilégiée, poussant à l'extrême le raisonnement selon lequel « quand on sait, on fait, quand on ne sait pas, on enseigne et quand on ignore, on forme les enseignants ». Ce qui me conduira, dans un second temps, à explorer quelques pistes largement fréquentées dans le cadre d'un enseignement très ancien (le plus ancien avec la philosophie ?) mais aussi à mener une réflexion sur la place de l'antiquité dans notre quotidien. Je terminerai par une anticipation sur le développement des ces deux disciplines, anticipation que l'on voudra bien me pardonner puisque je m'inscrirai dans des inspirations diverses sur ce qu'elles vont devenir dans la société et dans l'École qui poursuit sa longue histoire depuis l'Académie de Platon jusqu'aux virtual schools dont l'avenir n'est pas assuré à mon sens.

\section{1 - De quelques liens paradoxaux entre les langues, littératures et civilisation anciennes et les sciences de l'éducation}

\section{1. - L'émergence de la pédagogie en Grèce antique}

\footnotetext{
${ }^{1}$ ÉTIENNE (1997).

${ }^{2}$ Les sciences de l'éducation sont nées deux fois en France : au singulier en 1887 (GAUTHERIN, 2002) à la Sorbonne (chaire occupée par Henri Marion puis Ferdinand Buisson et Émile Durkheim qui l'abandonne pour fonder la sociologie) et au pluriel entre 1967 et 1970 avec trois universités (Bordeaux, Caen et Paris) rejointes par Lyon et Toulouse.
} 
La première trace d'une activité pédagogique se lit chez Socrate qui « corrompt la jeunesse » mais qui définit un projet et une pratique pédagogiques, la maïeutique. Cette référence au métier de sa mère lance déjà le débat entre les deux conceptions de l'enseignement qui s'affrontent encore de nos jours sous la forme d'une transmission directe des savoirs dénoncée comme une supercherie ou d'un « accouchement» de l'esprit de l'apprenant ${ }^{3}$. Dans le Ménon, le jeune esclave construit littéralement le théorème de Pythagore et les questions guidantes de Socrate correspondent à ce que l'on pourrait appeler, non sans quelque anachronisme, une situation-problème ${ }^{4}$ : refuser de se prendre au mirage du cours magistral considéré comme la transmission parfaite. Estimer au contraire que tant que la personne n'a rien fait du savoir, on ne peut ni le lui reconnaître ni inférer le moindre savoir utiliser ce savoir, ce que Le Boterf ${ }^{5}$ a défini comme étant la compétence dont il a fait un « attracteur étrange ».

\section{2 - Du sens de l'autorité et de l'autorité du sens dans l'éducation}

L'éducation peut être mortelle pour les individus, car elle n'est pas forcément libératrice, comme elle peut l'être pour les sociétés, mais elle peut aussi aider à grandir; nous savons que c'est là l'étymologie de l'auctoritas oubliée de bien des pourfendeurs du « laxisme ambiant $»^{6}$ : éduquer n'est rien d'autre que faire grandir, faire croître (de augeo), et l'auctor est celui qui autorise, au sens que lui ont donné les psychanalystes : la transgression réglée, libératrice, est l'horizon de l'être humain et la société n'a progressé que par celles dont Edipe, par exemple, fut, à son corps défendant, la première victime. Énigme du savoir mais triomphe de la pensée dans sa réponse au Sphinx, tragique de l'ignorance dans son parricide et dans l'inceste commis avec sa mère. Si l'on veut éduquer une personne (je reviendrai sur ce mot aussi), il est préférable de l'aider à grandir plutôt que de multiplier les interdits. À ces derniers incombe le rôle essentiel de la préservation, rôle qu'on ne saurait négliger dans la tradition humaniste qui reconnaît à l'enfant un certain droit à la sécurité pour se retrouver dans l'insécurité de l'apprendre. Mais l'autorité relève, elle, d'une ambition bien plus élevée ${ }^{7}$ car elle se construit et s'accepte sans violence physique ni verbale.

\section{3 - La création de l'école et ses métamorphoses}

$\mathrm{Ni}$ les mots ni les concepts ne sont innocents et la skhôlé ou le ludus grammaticus fournissent à l'école une identité première de téménos qui a pu provoquer de la confusion chez celles et ceux qui proposent de la sanctuariser. Il est vrai que la nuance n'est pas toujours facile à déterminer entre un lieu fait pour apprendre, qui va inexorablement déclencher de l'angoisse par perte de repères installés dans le long terme, obstacles à « la formation de l'esprit scientifique ${ }^{8}$, et un cocon qui serait tissé pour isoler l'enfant d'une société en perpétuel mouvement. C'est ce que n'a pas su comprendre, à mon sens, Illich $^{9}$ quand il a souhaité « déscolariser » ou « désinstitutionnaliser » la société. Celles et ceux qui rejettent les sciences de l'éducation et argumentent de bonne foi sur la nécessité d'en finir avec l'école ${ }^{10}$ effectuent toujours le même chemin. Il consiste à s'appesantir sur les défauts d'une école qui a marqué de son sceau les trois derniers siècles : il s'agissait d'apporter le savoir minimal à tous et de sélectionner les meilleurs. On est alors loin de l'idéal de l'honnête homme ; ce qu'on peut reprocher surtout à ce système « à deux vitesses », c'est que les femmes en sont écartées et qu'il leur est interdit de se montrer « savantes ». Ce modèle lassallien, inventé et mis au point au dix-septième siècle, a connu un fort développement aux dix-huitième et dix-neuvième siècles puis une longue agonie pendant la deuxième moitié du vingtième, confronté qu'il était

\footnotetext{
${ }^{3}$ ROBERT (2001).

${ }^{4}$ PERRENOUd (1999).

${ }^{5}$ LE BOTERF (1994).

${ }^{6}$ BRIGHELLI (2006).

${ }^{7}$ ROBBES (2013).

${ }^{8}$ BACHELARD (1995).

${ }^{9}$ ILLICH (1971).

${ }^{10}$ Durpaire / Mabilon-Bonfils (2014).
} 
à la contradiction d'une éducation pour tous avec une sélection des meilleurs qui ne se préoccupe pas (ou que trop peu) des moins bons.

1.4 - Une éducation visant l'égalité est-elle possible?

Comme finalement la massification l'a emporté, l'échec de la standardisation calquée sur un élève moyen ou, dans certains cas, sur l'élève excellent défini comme celui qui a les meilleures notes et les moyennes les plus élevées, a entraîné un changement radical. « Avec la massification scolaire amorcée dans les années 1960 et $1970^{11}$, et la possibilité offerte, en principe, à tous les élèves d'accéder aux formations les plus longues et les plus prestigieuses, le maintien des inégalités sociales face à l'école apparaît comme une sorte de scandale » écrit Dubet $^{12}$ au début du $21^{\text {ème }}$ siècle. Mais alors, que faire ? Comment inventer, ou réinventer, une éducation susceptible de mettre fin aux inégalités sociales ? Et le défi va bien au delà car, si l'on reprend les analyses de Boudon ${ }^{13}$, il y aurait une part de stratégie dans les choix effectués par les élèves et les familles. Les sciences de l'éducation et la sociologie de l'école ont fait l'effort de compréhension de la mécanique de reproduction mise en évidence par Bourdieu et Passeron ${ }^{14}$. Enfin, elles ont tenté de la nuancer et de la combiner avec le projet personnel de l'élève ${ }^{15}$ dans la lignée de la première loi d'orientation sur l'école. Ce n'est plus seulement l'égalité qui est visée mais l'épanouissement personnel.

\section{5 - Individualiser ou personnaliser? Une réponse soufflée par le théâtre antique}

Autrement dit, la question du développement de la société par la mise en concurrence des meilleurs a fait long feu et la promotion de l'individualisation, consécutive aux travaux béhavioristes des années $1950^{16}$, s'est heurtée à l'erreur fondamentale de cette école pour laquelle la décomposition en éléments simples et le respect du rythme de celle ou celui qui apprend sont les clés d'un enseignement réussi. Une fois de plus, des travaux sont venus apporter un correctif à une vision trop mécaniste de l'être humain et, pour ne citer qu'un auteur emblématique, c'est la théorie des «intelligences multiples » de Gardner ${ }^{17}$ qui résume le mieux la nécessité de passer de l'individualisation à la personnalisation. C'est encore à l'antiquité, mais cette fois aux Étrusques, que nous devons le mot : la personna désigne le masque que portaient les acteurs. Il y a deux avantages à s'inscrire dans cette mouvance : le jeu social ressemble fort à celui du théâtre où l'illusion de la transparence est vite balayée car nul n'est tenu de se dévoiler et le respect de l'intimité est un droit de toute personne. Mais il y a aussi cette subtile technique qui consistait à amplifier la voix et à donner davantage la parole à celui qui la prenait et les rôles de femmes étaient tenus par des hommes malgré l'Assemblée des femmes qui tenta vainement de revenir sur cet état de fait.

\section{6 - Catharsis et mimesis pour passer de l'enfance protégée à l'action}

C'est aussi au théâtre antique qu'on doit la catharsis (terme qui a été repris par les Grecs à la fin des années 2000 quand ils ont eu à dénommer les privations qu'ils endurent encore actuellement). Cette catharsis est traditionnellement associée à une purification mais on peut aussi la considérer comme une purgation ${ }^{18}$ :

La tragédie [...] est une imitation faite par des personnages en action et non par le moyen de la narration, et qui, par l'entremise de la pitié et de la crainte, accomplit la purgation des émotions de ce genre.

\footnotetext{
${ }^{11}$ Le lecteur comprendra que Dubet ne parle que de la massification du secondaire, porte d'entrée dans le supérieur, dont le modèle est le lycée calqué sur les collèges jésuites par Napoléon en 1802 ; le primaire, «l'école de la république », lui a été massifié très tôt en France, reprenant le modèle des écoles paroissiales.

${ }^{12}$ Dubet (sd).

13 BOUDON (1973).

14 BOURDIEU / PASSERON (1970).

${ }^{15}$ ÉTIENNE / BALDY / BENEDETTO (1992).

${ }^{16}$ SKINNER (1958).

${ }^{17}$ GARDNER (2008).

${ }^{18}$ ARstT, Poet., 1449 b28.
} 
Pour ma part, et dans une perspective éducationnelle, je préfère y voir une mimesis c'est-àdire une représentation de l'action qui permet d'éviter de se fourvoyer et qui retrouve, de manière assez inattendue, les mondes virtuels que le développement de l'informatique permet de créer avec de plus en plus de réalisme. Autrement dit, éprouver phobos (de la crainte) et éléos (de la pitié), ce qui est le but et le propre de la tragédie, revient à trouver le chemin de l'éducation vécue comme une libération, une émancipation. Ne pas en éprouver revient à rester dans le monde de la caverne et de ses illusions ${ }^{19}$; or, c'est précisément la mission de l'éducation que de réinscrire « les enfants de Gaïa » dans l'histoire de l'humanité pour qu'ils $\mathrm{y}$ inscrivent leur histoire personnelle ${ }^{20}$. Les mythes développés par les anciens sont devenus des ressources non seulement pour les psychanalystes mais aussi pour des éducateurs en proie au refus d'apprendre d'élèves qui préfèrent la force brutale de héros modernes et factices à la subtilité d'une pensée. Boimare ${ }^{21}$ fait grandir les garçons (surtout) qui sont « empêchés de penser » en les réinsérant dans les peurs qu'ils n'ont pu éprouver au cours de leur petite enfance à l'aide du mythe d'Héraklès/Hercule condamné à ses douze travaux pour avoir tué ses enfants. Nous retrouvons là les effets d'une éducation qui confronte les enfants à une réalité médiée par le langage mais aussi par les images de l'antiquité, aujourd'hui source d'inspiration pour les créateurs de jeux informatiques.

\section{7 - De l'invention de la didactique et de son succès}

Si la pédagogie plonge ses racines dans l'antiquité, il n'en va pas de même pour la didactique. En effet, le pédagogue se soucie avant tout de l'enfant et de son éducation tandis que le didacticien (ou la didacticienne) va avoir pour cible première le contenu de ce qu'il ou elle enseigne. Ainsi abandonnera-t-il volontiers le projet d'éduquer en se contentant de celui d'instruire. Mais la didactique a eu trois vies. La première est ainsi définie par Komenský, qui a latinisé son nom en Comenius : Ars omnibus omnia omnino docendi (la technique pour tout enseigner à tous en totalité). Tel est le projet avoué de la Magna Didactica en 1657 ; aujourd'hui, les ambitions des didactiques sont plus modestes (ou plus concrètes) et se contentent de partir des « savoirs savants ${ }^{22}$, voire des «pratiques sociales de référence ${ }^{23}$ tandis que la didactique professionnelle s'intéresse à la manière dont s'exercent les métiers et à la formation pour y préparer ${ }^{24}$. On comprendra que dans ce type d'approche finalement assez technologique, les apports de l'antiquité se soient limités à clarifications linguistiques : ainsi, il n'existe pas (ou plus) de didactique générale mais un champ se structure autour de la didactique comparée, puisque le fait d'enseigner ne peut se réduire à une seule variable qui serait la discipline de référence. Si l'on parle de sciences de la formation, le divorce avec l'antiquité est totalement consommé car la transmission orale entre esclaves n'a pas permis de profiter des savoirs professionnels déjà bien présents mais peu valorisés par des écrits. Qu'on se rappelle la difficulté à exécuter les recettes d'Apicius ou que l'on se réfère à l'archéologie expérimentale qui essaie de retrouver les gestes de métier à partir du seul résultat en architecture terrestre ou navale.

\section{8 - Deux disciplines également menacées pour des raisons diamétralement opposées.}

Aujourd'hui, les langues et cultures de l'antiquité ainsi que les sciences de l'éducation (et de la formation, aurait-on dû ajouter) sont l'objet d'attaques multiples et variées, similaires à celles que subissent les humanités, alors que Calame ${ }^{25}$ les montre pour ce qu'elles sont : des sciences humaines. Les deux disciplines ont pu prêter le flanc à des critiques dont certaines

\footnotetext{
${ }^{19}$ LANDOEUER (2013).

${ }^{20}$ TOZZI (2012).

${ }^{21}$ BoIMARE (1999).

${ }^{22}$ Chevallard (1985).

${ }^{23}$ MARTINAND (1986).

${ }^{24}$ PASTRE (2011).

${ }^{25}$ CALAme (2011).
} 
m'apparaissent justifiées : les premières pour n'être finalement qu'une occasion de manifester la « distinction » chère à Bourdieu ${ }^{26}$ et les secondes pour ne pas offrir de perspectives claires en vue d'une application systématique de leurs préceptes ${ }^{27}$. Les attaques contre les sciences de

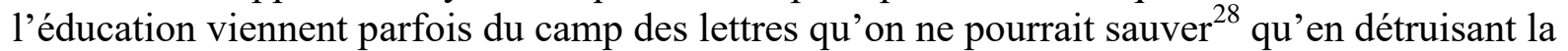
formation des professeurs du secondaire, d'ailleurs constamment remise en question par les ministres, aujourd'hui sous couvert de «mastérisation ». Ces réformes inspirées par le processus de Bologne (1999) aboutiront-elles à un renouveau du compagnonnage pratiqué par des anciens soucieux de faire partager leur expérience (mais pas leurs classes ?), à un acharnement sur certains savoirs (grammaticaux ?) et à l'oubli du patrimoine à transmettre ? Or ce patrimoine continue à se développer au rythme de l'élévation du niveau moyen constaté depuis un bon siècle ${ }^{29}$ et ce serait une contre-vérité que de l'oublier. Ces deux disciplines (LCA et sciences de l'éducation) ont donc intérêt à faire front commun dans une perspective de démocratisation de l'enseignement et de contribution des langues et cultures de l'antiquité à cette entreprise.

\section{2. - De nouvelles méthodes et de nouveaux enseignements en lettres avec une place renouvelée de l'antiquité dans un monde en quête de repères}

\section{1 - Partir sans trahir pour mieux revenir}

Nombreux sont ceux qui connaissent le début de cette citation de Jean Jaurès : « Un peu d'internationalisme éloigne de la patrie ; beaucoup d'internationalisme y ramène. Un peu de patriotisme éloigne de l'Internationale ; beaucoup de patriotisme y ramène ». Je partirai donc de la volonté que j'ai exprimée en 1982 quand j'ai été élu président de l'Association pour la Recherche dans l'Enseignement des Langues Anciennes Montpellier (ARELAM) qui se fondait alors : associer recherche et enseignement des langues anciennes. Cette association a par la suite adhéré à la Coordination Nationale des Associations Régionales ${ }^{30}$ des Enseignants de Langues Anciennes (CNARELA), elle-même fondatrice d'Euroclassica qui regroupe des associations dans vingt-six pays autour du projet et des valeurs portées par une vision humaniste de l'antiquité, de ses langues et de ses cultures. Je m'inspirerai aussi de trois thèses de doctorat :

1. la mienne sur la didactique des langues anciennes (soutenue en 1996 et publiée en 1997) qui m'a valu un message amical de Ghislaine Viré ; elle part de l'idée que la recherche en didactique des disciplines (en l'occurrence celle des langues anciennes) ne doit pas se limiter à l'individu-élève mais gagne à se préoccuper de la personne qui apprend dans sa globalité et son milieu, ce qui inclut la «famille », bien sûr, mais aussi l'établissement et son « effet-établissement » ainsi que le libre choix de la discipline dans la perspective d'une éducation à l'orientation. Si j'avais alors connu la didactique professionnelle, je lui aurais ménagé une large place à côté et en interaction avec les autres types de recherche car ce qui m'intéressait (et qui m'intéresse encore) est de comprendre comment les élèves peuvent choisir d'apprendre les LCA, y trouver du plaisir et se développer grâce à elles,

2. celle de Charlotte Vanhalme ${ }^{31}$ intitulée Citoyenneté postmoderne et didactique des langues anciennes : quel projet d'autonomie intellectuelle pour l'apprenant? Elle

\footnotetext{
${ }^{26}$ BOURDIEU (1979).

${ }^{27}$ PERRENOUd (2004).

${ }^{28}$ Je fais ici allusion à une association « Sauver les lettres » et à son site http://www.sauv.net/ qui ont trouvé un ennemi à pourfendre « les pédagogistes »; lesquels, à l'instar de Socrate, corrompraient les jeunes, l'enseignement et les lettres !

${ }^{29}$ Il n'y avait qu'un pour cent de bacheliers en France en 1900 et ils furent $63 \%$ en 2000 . Aujourd'hui, l'objectif français est d'une personne sur deux dans une génération à bac+3.

${ }^{30}$ Le mot « recherche » pour traduire la deuxième initiale du sigle est une spécificité de l'association montpelliéraine.

${ }^{31}$ VANHALME (2011).
} 
s'est donné pour objectif d' " envisager de manière critique les différents scénarios susceptibles d'aboutir au développement de l'autonomie intellectuelle et à l'acquisition des concepts de l'éthique citoyenne par l'apprenant »,

3. et celle de Dominique Augé ${ }^{32}$ publiée sous le titre Refonder l'enseignement des langues anciennes, le défi de la lecture. L'auteure pose le principe d'une refondation d'un enseignement auquel elle a déjà beaucoup participé dans le cadre du projet Helios qui a donné naissance à une plate-forme de référence pour les langues anciennes $^{33}$. Une nouvelle fois, l'objectif de cette recherche est présenté avec clarté mais aussi conviction fondée sur de l'expérience et des travaux de recherche menés sous la direction de Françoise Létoublon : «réfléchir à la spécificité du champ disciplinaire pour en déduire des principes et une pratique pédagogique, largement appuyée sur l'intégration des technologies modernes, suffisamment cohérente et efficiente pour amener les étudiants à l'appropriation d'une culture par l'assimilation d'une langue ».

Finalement, ces trois thèses ont en commun de tirer d'une longue histoire des perspectives pour un projet de l'apprenant et de l'enseignant (en tant que personnes et pas seulement latiniste, helléniste ou professeur de langues anciennes). Elles se complètent en ce qu'elles envisagent des évolutions didactiques pour démocratiser cet enseignement (Étienne), éduquer des citoyens (Vanhalme) et les instituer comme lecteurs (Augé). C'est une belle illustration de la pensée de Jaurès : partir des langues et cultures de l'antiquité pour mieux y revenir mais aussi partir des savoirs en éducation pour mieux retrouver le bonheur de l'étude.

2.2 - Et si l'objet de cet enseignement, c'était le sujet ou la personne?

Renouveler l'enseignement des lettres, des langues et cultures anciennes et modernes, bref les humanités, ne peut se concevoir sans une réflexion ${ }^{34}$ sur ce qu'il faut enseigner (les programmes ou, forme étrangère à la France, mais de plus en plus reprise par ses chercheurs et ses administrateurs de l'éducation, le curriculum), comment l'enseigner (la didactique) et la manière d'amener les élèves à l'apprendre (la pédagogie). C'est sans doute la notion de contrat didactique due à Brousseau ${ }^{35}$ qui convient le mieux à cette entreprise : Jacques Brel dans sa chanson Rosa (1962) et Marcel Pagnol dans ses souvenirs d'enfance (1960) ont démontré à l'envi les effets contre-productifs d'un enseignement mécanique qui part de l'acquisition d'un savoir « par cœur » pour permettre, quelques années plus tard et pour ceux qui n'ont pas abandonné en cours de scolarité ${ }^{36}$, l'accès à la beauté des Bucoliques dont le petit-fils d'immigré espagnol a donné une traduction poétique (1958). L'élève n'est plus aujourd'hui un objet qui se contente d'enregistrer et d'exécuter les instructions du maître. Le développement de différentes approches, notamment celle de la psychologie du développement, et l'évolution de notre société l'ont bien fait passer du statut d'infans (celui qui n'a pas le droit à la parole) au rôle institutionnel mais aussi personnel de discipulus (celui qui est là pour apprendre, l'apprenant comme le nomme si bien Vanhalme). Les quolibets des nostalgiques de l'élève ne parviendront pas à nous faire abandonner cette belle métaphore du disciple. Le terme fait un peu suranné et il peut se rapporter à une emprise dont les sectes ont

\footnotetext{
${ }^{32}$ Auge (2013).

${ }^{33}$ Le site http://helios.fltr.ucl.ac.be/ est hébergé par l'université catholique de Louvain à Louvain la neuve. Il s'agit d'une réalisation remarquable qui présente aussi bien des séquences d'enseignement que des suggestions didactique. Le projet date de 2005 et certains aspects mériteraient d'être revus au regard du développement des possibilités d'internet et des développements didactiques en sciences de l'éducation mais je considère ce travail collectif comme une anticipation sur les MOOC (massive open online course ou cours gratuits en ligne destinés à un grand public) qui sont devenus au début des années 2010 un modèle peu remis en cause par le monde de l'enseignement.

${ }^{34}$ Altet / Desjardins / Étienne / PAQUAy / PERRENOUd (2013).

${ }^{35}$ BROUSSEAU (1990).

${ }^{36}$ CIBOIS (2011).
} 
le secret. C'est donc le terme d'apprenant qui nous semble le plus précis pour désigner un sujet ou une personne en pleine activité... d'apprentissage ! Qu'en est-il alors du maître ? 2.3 - Le choix du maître entre l'asymétrie des savoirs et la dissymétrie des pouvoirs «Enseigner est un métier qui s'apprend » tel est l'accord que l'opération de mastérisation a réussi à réaliser et qu'a entériné le numéro 435 des Cahiers pédagogiques $^{37}$; aujourd'hui, treize ans plus tard, le constat est le même et il est même constaté que c'est « un métier qui bouge $»^{38}$. Or, tout ce qui s'apprend peut et doit faire l'objet de recherches sur ce savoir, de recherches en didactique également, surtout si les savoirs savants et professionnels «bougent ». Les progrès de la didactique comparée permettent d'envisager la mise en évidence d'invariants ainsi que la présence de spécificités liées à chaque discipline mais aussi à chaque groupe de disciplines. Les langues et cultures de l'antiquité appartiennent au groupe des langues et civilisations et les enseigner sans étroite concertation avec les langues dites vivantes ne permettrait pas de les situer dans un continuum dans lequel les élèves ont tout intérêt à se créer des repères et des références pour élaborer leur projet d'études ou de choix optionnels. Le rôle de l'enseignant se situe plutôt dans la tradition de Sophocle et d'Euripide qui ont privilégié la présence de plusieurs acteurs sur scène que dans celle d'Eschyle proche de la tradition qui ne mettait en présence qu'un acteur et le chœur. Cette formation et cette transformation des enseignants donnent une belle chance aux enseignants de LCA qui ont su, nous l'avons évoqué pour Helios, s'emparer d'outils informatiques et d'internet pour leur enseignement. La personnalisation des apprentissages et de l'enseignement ne peut se concevoir sans un travail d'équipes de classe, de discipline, d'établissement. Elle se complète utilement par un engagement dans la promotion de la discipline au service d'une démocratisation qui repose sur l'épanouissement des personnes et le développement de la société.

\section{3. - Promouvoir l'enseignement des langues et cultures de l'antiquité en les faisant contribuer à l'épanouissement des personnes et au développement de la société.}

Je terminerai ce chapitre en développant quelques idées iconoclastes, voire utopiques, issues des travaux de la CNARELA dont la dimension nationale m'inspire sans que j'aie le moins du monde l'intention d'oublier son rayonnement international à travers sa participation déjà évoquée à Euroclassica et de recherches collectives ou personnelles. Les journées des Assises des Lettres organisées à l'université Toulouse-Le Mirail sous la houlette de Jean-Claude Carrière les 27, 28 et 29 mai 2010 ont été également un moment fort dans mon engagement sur cette réflexion bi-disciplinaire puisque c'est à cette occasion que j'ai commencé à la formaliser.

\section{1. - Toujours penser aux origines pour se projeter}

En France, les Associations régionales d'enseignants de langues anciennes (ARELA) se sont créées à partir de celle de Besançon et de ses fameuses thèses ${ }^{39}$ en référence aux travaux de Bourdieu et Passeron ${ }^{40}$ sur la reproduction et avec une volonté de démocratisation qui manifestait une rupture franche avec la Franco-ancienne ${ }^{41}$ plus corporatiste et qui ne se préoccupait ni du recrutement social des élèves ni de leur devenir dans une société alors en plein essor. On était à la fin des Trente Glorieuses (1945-1973) et au début de la massification qu'allait consacrer, en France, la réforme Haby qui créait le «collège unique » en 1975 avec l'intention de faire entrer dans le secondaire tous les enfants scolarisés : je rappellerai qu'en

\footnotetext{
${ }^{37}$ ÉTIENNE / ZAMARON (2005).

${ }^{38}$ AMIEL / MEVEL (2014).

${ }^{39}$ ARELAB (1979).

${ }^{40}$ BOURDIEU / PASSERON (1970).

${ }^{41}$ Il s'agit de l'association des professeurs de lettres classiques qui enseignent en France les trois disciplines que sont le français, le latin et le grec. À l'époque, cette association ne visait pas la démocratisation de l'enseignement secondaire, d'où la scission évoquée ci-dessus.
} 
1900, la France comptait 1\% de bacheliers ; il n'y a jamais eu autant de latinistes et d'hellénistes qu'aujourd'hui ${ }^{42}$ ! Mais moins de $10 \%$ d'entre eux passent les épreuves du baccalauréat dans ces disciplines (ibid.) ! C'est un problème qui se retrouve dans bien d'autres pays : les élèves qui font le choix des langues anciennes développent des talents de structuration de la pensée et la qualité de leur expression, la clarté de leur communication mais ils sont trop souvent placés devant le dilemme du choix d'études littéraires dévalorisées (aux yeux de trop nombreuses personnes, pas aux miens) ou d'études scientifiques survalorisées, tout au moins dans le secondaire français. Il convient de desserrer cette étreinte qui néglige le fait que des personnalités comme celle de Paul Valéry ont établi qu'il était inutile et stupide de se priver d'une voie ou de l'autre.

3.2 - Se référer à une approche méditerranéenne du monde Vanhalme a montré dans sa thèse ${ }^{43}$ qu'il est essentiel de former un citoyen grâce à l'enseignement des LCA. Dans le sillage de Perrenoud ${ }^{44}$ et de l'équipe de recherche du laboratoire de recherche de l'université de Genève, je voudrais réaffirmer l'importance politique de l'étude des LCA. Elles facilitent la rencontre des fondements premiers de la démocratie, voire encouragent à critiquer certains de ses éléments fondateurs comme le sort réservé aux esclaves et aux femmes. La réflexion sur la notion de citoyen et celle de métèque (au sens étymologique) fait évoluer les références vers des approches moins ethnocentrées. L'enseignement et l'apprentissage des langues anciennes ont tout à gagner à se référer à Carthage et à Troie, à l'Autre, qu'il soit différent ou semblable ! Les historiens, les spécialistes des mythes et de l'anthropologie peuvent nous aider faire évoluer nos représentations et celles des élèves vers une conception d'un monde qui était déjà multipolaire et les références gréco-latines traditionnelles, qui se limitent à quelques années et à quelques auteurs, s'étendent aujourd'hui à l'ensemble de la Méditerranée, voire à une conception « circulaire » du monde, et couvrent pratiquement vingt siècles. À un niveau très pratique, le simple fait de pratiquer cet enseignement en adoptant les tria nomina pour renommer les élèves, pratique courante en anglais, permet de leur faire découvrir qu'Afer ou Niger désignaient des personnes et des gentes qui n'avaient pas la pureté des premiers Romains... qui n'étaient d'ailleurs pas des modèles de pureté !

3.3 - Remettre l'analyse à sa place

Il en va de même pour l'enseignement de la langue qui a été pendant trop longtemps fondé sur l'analyse à l'instar de celui de la musique qui imposait le solfège d'abord. Il ne s'agit pas de pratiquer le latin vivant comme dans les Feria Latinae qui se sont déroulées à Nice jusqu'en 1998 et se tiennent depuis 2000 à l'abbaye Saint-Michel-de-Frigolet, près de Tarascon ni de l'apprendre «sans peine » avec la méthode Assimil rédigée par l'ingénieur Desessard ${ }^{45}$. C'est au contraire un appel à une approche résolument linguistique que je répercute. Elle s'appuie sur les travaux déjà anciens de Claude Fiévet ${ }^{46}$ ou le cours de Jean-Noël Michaud ${ }^{47}$. Sans tomber dans les outrances des méthodes comportementalistes des années 1960, ces auteurs et d'autres ont tenté de faire découvrir la langue latine de manière audio-orale d'abord, puis par observation et enfin d'articuler l'analyse, la compréhension des textes et la production d'énoncés. Au terme d'un semestre universitaire, les étudiantes et étudiants " grands débutants » assidus sont en mesure de rédiger un récit en langue latine. C'est ainsi que nous apprenons les langues secondes et l'analyse ne se justifie que quand elle permet de comprendre ce qu'on n'avait pas compris puis de construire une grammaire de la langue

\footnotetext{
${ }^{42}$ CiBOIS (2011).

${ }^{43}$ VANHALME (2011).

${ }^{44}$ PERRENOUD (2003).

45 DESESSARD (1966).

${ }^{46}$ FIEVET (1971).

${ }^{47}$ MiChAUd (2001).
} 
apprise en remplacement de la «grammaire implicite » que nous a inspirée la pratique de notre langue première. Il est plaisant pour un universitaire de constater que les obstacles à l'apprentissage du latin diffèrent pour des Allemands, des Espagnols et des Français, d'où la nécessité d'un recours à l'inter langue pour comprendre d'où viennent les obstacles à l'apprentissage. Ainsi, l'ordre des mots dans la phrase française est-il le principal obstacle à lever pour les étudiants français.

3.4 - Renouveler les manuels, les supports et les situations d'apprentissage

Dès lors, renouveler les manuels et tous les supports d'enseignement (notamment informatiques, comme le projet Helios et le site de Philippe Cibois) devient une urgence qui n'est toujours pas traitée à la hauteur des espérances des enseignants ou des chercheurs en didactique des langues anciennes. Si quelques efforts ont été faits, le renvoi à des manuels de « grands débutants » fait dans le paragraphe précédent montre, tout au moins pour la France, une dépendance par rapport aux programmes qui impose toujours un lien plus ou moins explicite avec une inspectrice ou un inspecteur, même si leur droit à co-signer des ouvrages a été considérablement réduit. La présence de chercheurs, comme dans la collection supervisée par Pierre Grimal, n'a pas toujours constitué l'antidote à cette dérive conformiste car le recrutement d'un universitaire ne garantit pas sa capacité à diriger une œuvre collective comme une collection de manuels. C'est là qu'une conjonction entre des praticiens de l'enseignement et des chercheurs qui ont encore une activité en collège ou en lycée, comme cela est le cas pour Dominique Augé ${ }^{48}$, devrait induire une sortie de l'impasse actuelle dans laquelle les manuels ne progressent que trop lentement en fonction d'une évaluation par les utilisateurs que sont les élèves et les enseignants.

3.5 - Enseigner les langues et cultures de l'antiquité de la façon la plus vivante possible Comment faire connaissance avec la vie antique sans aller sur les lieux, sans développer encore et toujours les voyages scolaires en cours d'études pour y éprouver les conseils de Claude Hagège ${ }^{49}$ et de Jacques Lacarrière ${ }^{50}$ ? Ces promoteurs d'un rapport vivant à la langue se fondent tous deux sur le voyage pour comprendre la langue et la culture. Ils sont partisans de la découverte évoquée au paragraphe ci-dessus. Ainsi préservée, l'émotion de la rencontre ne manque pas de déclencher une volonté d'approfondissement qui procure du travail à l'élève mais un travail fondé sur le désir d'apprendre. Oser le théâtre en latin (avec une pièce rédigée par les élèves, apprise par eux dans la langue de Plaute et Térence, puis représentée devant une assemblée de parents), rédiger des romans dans cette langue et faire vivre toutes sortes d'événements qui promeuvent les langues anciennes dans les établissements, dans un esprit de coopération et non de compétition avec les autres disciplines, telles sont les pratiques que bien des chercheurs ont eu l'occasion de répertorier, d'étudier et d'évaluer avec leurs auteurs pour les améliorer.

\section{6 - Souligner l'omniprésence de l'antiquité}

Ces sorties scolaires mais aussi ces entrées dans l'établissement de personnes ressources, bref, ces échanges entre le monde scolaire et le monde contemporain sont destinés à souligner l'omniprésence de l'antiquité déjà mise en évidence par Jacques Gaillard ${ }^{51}$. Tout peut servir de prétexte pour développer une pédagogie de projet : les élèves repèrent dans un texte du quotidien les mots issus du latin, du grec mais aussi de l'arabe ou de l'anglais. Ils en dressent ensuite l'évolution et développent ainsi leurs contacts avec la complexité de la linguistique. Mais le défi consiste surtout à leur faire inventer des projets autour de la présence de l'antique dans le monde actuel, à l'instar de Célestin Freinet qui a fait du texte libre le modèle de ce qu'il a dénommé sa «méthode naturelle », alors qu'elle l'est si peu !

\footnotetext{
${ }^{48}$ Auge (2013).

${ }^{49}$ HAGEGE (2013).

${ }^{50}$ LACARRIERE (2013).

${ }^{51}$ GAILLARD (1993).
} 


\section{7 - De l'élève en latin ou grec au citoyen de demain}

$\mathrm{Au}$ lieu des les opposer, comme le font constamment les tenants de deux camps qui prônent les savoirs sans leur application (approche trop abstraite) ou les savoir-faire sans les savoirs (approche trop pragmatique), ne pourrait-on réfléchir à des tâches qui soient à la fois littéraires et propices au développement de compétences transversales (les comptes rendus, les critiques, les recensions, les analyses, les discours ou encore le récit en langue ancienne) ? Enseigner les langues et cultures de l'antiquité n'impose pas, bien au contraire, de se retirer dans un espace clos et aseptisé. Elles fournissent une occasion rêvée pour mener une réflexion sur des écarts entre ce qui était vivant (c'est pour cela que « langues mortes » est une appellation inappropriée), ce qui l'est et ce qui le deviendra pour peu que des humains joignent leurs efforts dans le cadre de projets. C'est l'occasion de rappeler, après Jean-Louis Dumortier $^{52}$, que l'intention est de concevoir et de pratiquer, au sein des classes et des établissements, grâce à une coopération entre enseignants et chercheurs en éducation, " une rénovation d'esprit humaniste orientée par la double perspective d'un "mieux penser" et d'un "mieux faire" ». L'instruction, définie comme la transmission des savoirs, n'est pas « soluble » dans l'éducation; il faut y ajouter et combiner la formation professionnelle (y compris l'orientation), l'émancipation de la personne et son épanouissement comme citoyen, ce que nous avons tenté de définir comme « projet personnel de l'élève ${ }^{53}$. 3.8 - Ouverture sur un renouvellement de nos enseignements grâce à la recherche Pour être conforme à mes principes et intentions, je laisserai volontiers les lecteurs de ces mélanges ajouter leurs propres idées en vue du renouvellement de l'enseignement des langues, lettres et cultures de l'antiquité sous forme de projet personnel et/ou collectif qui donnera lieu à des recherches-action, voire, ce que je préfère, à des recherches sur l'action de les transmettre ! Nous avons essayé de créer ce type d'interface entre enseignants du secondaire et enseignants-chercheurs, Dominique Augé et moi ${ }^{54}$ (2013), en coordonnant un hors-série numérique d'une revue, les Cahiers pédagogiques qui entend « changer la société pour changer l'école, changer l'école pour changer la société » depuis la Libération en application du rapport Langevin-Wallon ${ }^{55}$, issu des demandes du Conseil national de la résistance et de sa volonté de démocratiser, au sens plein du terme, l'enseignement secondaire.

\section{Conclusion}

Nombreux sont les professeurs de renom (Jean-Pierre Vernant, Jacqueline de Romilly, André Mandouze, Maurice Lacroix, Jean-Claude Carrière, Jean-Louis Dumortier et Ghislaine Viré pour ne citer que ceux-là) ou plus humbles qui ont compris et appliqué le principe que l'on enseigne plus ce que l'on est que ce que l'on sait. Aujourd'hui et demain, les élèves et leurs familles ayant choisi les langues, littératures et cultures de l'antiquité peuvent et doivent rencontrer des enseignants qui les motivent par leur enthousiasme et leurs connaissances comme ils les forment grâce à leur compétence et à leur valeur humaine. J'ai tenté dans ce chapitre de montrer quelles ressources sont déjà exploitées par une discipline ancienne mais qui a su et sait évoluer grâce aux apports d'une discipline plus récente mais qui puise ses

\footnotetext{
${ }^{52}$ DUMORTIER (2014) p. 25.

${ }^{53}$ ÉTIENNE / BALDY / BENEDETTO (1992).

${ }^{54}$ Auge / ÉTIENNE (2013).

${ }^{55}$ LANGEVIN / WALLON (1947). Ce rapport, demandé en 1944 et remis en 1947, plus connu sou l'appellation de «plan Langevin-Wallon, ne connut pratiquement pas de mise en œuvre, même s'il inspira toutes les réformes éducatives de la fin du $20^{\mathrm{e}}$ siècle en France. Les « classes nouvelles », avec latin, travaux manuels et effectif limité à 25 élèves, en furent la seule application immédiate. Elles entendaient faciliter la démocratisation du secondaire en rompant avec la domination du cours magistral et les effectifs élevés qu'il suppose. Leur bulletin de liaison est devenu la publication des Cahiers pédagogiques qui est citée à plusieurs reprises dans ce chapitre et compte plus de cinq cents numéros.
} 
racines dans le monde grec classique ou dans la romanité étendue, les sciences de l'éducation. Ce qui peut les unir, c'est de faire œuvre et front communs, en s'appuyant sur cette longue durée de la civilisation, pour faire grandir les enfants, les instituer comme élèves mais aussi futurs travailleurs, citoyens en devenir et personnes épanouies. C'est la recherche sur cet enseignement qui pourra y contribuer, mais une recherche à parité entre enseignants du secondaire et enseignants-chercheurs, avec une belle part de créativité réservée aux élèves.

Références bibliographiques et sitographiques :

M. Amiel / Y. Mevel (2014), Enseignant, un métier qui bouge, Cahiers pédagogiques, dossier du n ${ }^{\circ} 514$, p. 12-57.

ARELAB (1979), Thèses de Besançon, supplément au Bulletin ARELAB n 14.

Aristote (1932, trad. J. Hardy), Poétique, Paris, Les Belles Lettres.

D. Auge (2013), Refonder l'enseignement des langues anciennes, le défi de la lecture, Grenoble, ELLUG, collection "Didaskein".

D. Auge, R. Étienne (2013, coord.), Apprendre les langues et cultures de l'Antiquité, Cahiers pédagogiques, Hors-série-numérique, $\mathrm{n}^{\circ} 32$.

G. BACHELARD (1955), La formation de l'esprit scientifique, Paris, Vrin.

S. BoImARE (1999), L'enfant et la peur d'apprendre, Paris, Dunod.

R. BOUdON (1973), L'Inégalité des chances. La mobilité des chances dans les sociétés industrielles, Paris, Armand Colin.

P. Bourdieu (1979), La Distinction. Critique sociale du jugement, Paris, éd. de Minuit. P. BourdieU, J.-Cl. PASSERON, (1970), La reproduction, éléments pour une théorie du système d'enseignement, Paris, éd. de Minuit.

J. BREL (1962), Rosa, Bruxelles : Fondation Jacques Brel.

J.-P. BRIGHELLI (2006), À bonne école, Paris, Jean-Claude Gawsewitch éditeur.

C. CAlame (2011, $2^{\mathrm{ème}}$ éd.), Mythe et histoire dans l'Antiquité grecque, Paris, Les Belles Lettres.

J.-Cl. CARRIERE (1987), Les mythes et les notions morales dans Les travaux et les jours d'Hésiode, thèse de doctorat d'Etat, université de Besançon.

Ph. CiBoIs (2011), L'enseignement du latin en France, une socio-histoire, texte inédit, décembre 2011, Uqac, les classiques des sciences sociales, téléchargé le 7 octobre 2014, http://dx.doi.org/doi:10.1522/030295563.

Cl. DeSESSARD (1996), Le latin sans peine, Paris, Assimil.

F. DUBET (sd), Éducation, sociologie de l'éducation, article du site de l'université de Mons, consulté le 7 octobre 2014, https://moodle.umons.ac.be/mod/resource/view.php?id=43173. J.-L. DuMORTIER (2014), Compétences, littérature et humanisme, conférence aux Assises des Lettres (Toulouse, 27-29 mai 2010), Puzzle, bulletin du CIFEN (Centre interfacultaire de formation des enseignants) ; $\mathrm{n}^{\circ} 33$, p. 20-27.

F. DuRPaire, B. MABILON-Bonfils (2014), La fin de l'école, l'ère du savoir-relation, Paris, PUF.

R. ÉTIENNE (1997). Didactique des langues anciennes, Recherches récentes sur leur enseignement dans les établissements secondaires français, Tendances et évolutions - Apports et perspectives, Lille, Presses du Septentrion.

R. ÉTIENNE, A. et R. BALDY, P. BenEdetTo (1992, $5^{\text {ème }}$ éd. en 2000, numérique en 2016), Le projet personnel de l'élève, Paris, Hachette Éducation, collection pédagogies pour demain, série nouvelles approches dirigée par Jean-Pierre Obin.

R. ÉTIENNE, A. ZAMARON, (2005, coord.), Enseigner, un métier qui s'apprend, Cahiers pédagogiques, dossier du $\mathrm{n}^{\circ} 435, \mathrm{p} .7-53$. 
Cl. FIEVET (1971,), Une expérience de latin audio-oral à l'usage des grands débutants, Vita Latina, $\mathrm{n}^{\circ} 42$, p. 13-19 et $\mathrm{n}^{\circ} 43$, p. 8-20.

J. Gaillard (1993), Beau comme l'Antique, Arles, Actes Sud.

H. GARDNER (2008), Les intelligences multiples, la théorie qui bouleverse nos idées reçues,

Paris, Retz.

J. GAUTHERIN (2002), Une discipline pour la république. La science de l'éducation en France (1882-1914), Berne, Peter Lang.

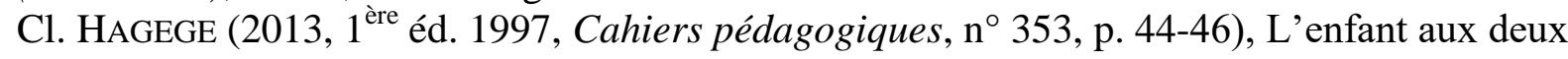
langues anciennes ? Cahiers pédagogiques, Hors-Série-Numérique, n 32, p. 24-27.

J. LACARRIERE (2013, reprise partielle d'un entretien de 1991), Invitation aux voyages,

Cahiers pédagogiques, Hors-Série-Numérique, $\mathrm{n}^{\circ} 32$, p. $22-23$.

F. LANDOEUER (2013), Socrate dans la cité, Paris, se $\mu$ eïon éditions.

Rapport LANGEVIN-WALLON, éd. Mille et une nuits, 2002, réédition commentée par Claude Allègre, François Dubet et Philippe Meirieu.

G. LE BOTERF (1994), De la compétence, essai sur un attracteur étrange, Paris, Les éditions d'organisation.

J.-L. MARTINAND (1986), Connaître et transformer la matière, Berne, Peter Lang.

J.-N. Michaud (2001), Le petit monde de Quartilla, Paris, l'espace d'un instant.

M. PAGnOL (1958, traduction de Virgile), Les Bucoliques, Paris, Grasset.

M. PAgnol (1960), Le temps des secrets, Monte-Carlo, éditions Pastorelly.

P. PASTRE (2011), La didactique professionnelle, approche anthropologique du

développement chez les adultes, Paris, PUF, collection Formation et pratiques

professionnelles.

Ph. PERRENOUd (1999), De l'analyse de l'expérience au travail par situations-problèmes en formation des enseignants, in $\mathrm{E}$. Triquet et $\mathrm{C}$. Fabre-Col (dir.), Recherche $(s)$ et formation des enseignants, Grenoble, IUFM, 1999, p. 89-105.

$\mathrm{Ph}$. PerRenoud (2003), L'école est-elle encore le creuset de la démocratie ? Lyon, Chronique Sociale.

Ph. PERRENOUd (2004), Les sciences de l'éducation proposent-elles des savoirs mobilisables dans l'action ? In Cl. Lessard, M. Altet, L. Paquay et Ph. Perrenoud (Eds), Entre sens commun et sciences humaines. Quels savoirs pour enseigner? Bruxelles, De Boeck, p. 139-157.

B. RoBBes (2013), L'autorité éducative, la construire et l'exercer, Amiens, scerén-CRDPCRAP-Cahiers pédagogiques, Repères pour agir.

A. RoberT (2001), Débat : Denis Kambouchner/Philippe Meirieu, Revue française de pédagogie, Volume 137, La pédagogie et les savoirs: éléments de débat, p. 5-16.

B.F. SKINNER (1958), Teaching machines, Science, n 128, p. 969-77.

M. Tozzi (2012), Nouvelles pratiques philosophiques. Répondre à la demande sociale et scolaire de philosophie, Lyon, Chronique sociale.

C. VAnhalme (2011), Citoyenneté postmoderne et didactique des langues anciennes : quel projet d'autonomie intellectuelle pour l'apprenant? Thèse de doctorat non publiée sous la direction de Ghislaine Viré, Bruxelles, ULB. 